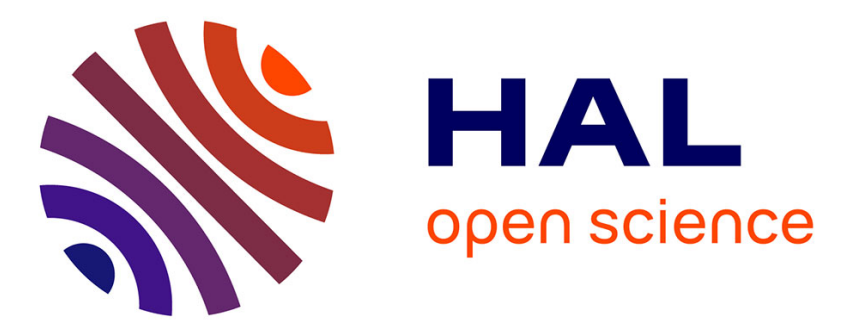

\title{
L'artiste faussaire et la création du réel: The Brooklyn Follies de Paul Auster
}

François Hugonnier

\section{To cite this version:}

François Hugonnier. L'artiste faussaire et la création du réel: The Brooklyn Follies de Paul Auster. Revue Française d'Etudes Américaines, 2012, 3 (133), pp.38-52. 10.3917/rfea.133.0038 . hal-03382967

\section{HAL Id: hal-03382967 \\ https://univ-angers.hal.science/hal-03382967}

Submitted on 18 Oct 2021

HAL is a multi-disciplinary open access archive for the deposit and dissemination of scientific research documents, whether they are published or not. The documents may come from teaching and research institutions in France or abroad, or from public or private research centers.
L'archive ouverte pluridisciplinaire HAL, est destinée au dépôt et à la diffusion de documents scientifiques de niveau recherche, publiés ou non, émanant des établissements d'enseignement et de recherche français ou étrangers, des laboratoires publics ou privés. 


\title{
L'ARTISTE FAUSSAIRE ET LA CRÉATION DU RÉEL : THE BROOKLYN FOLLIES DE PAUL AUSTER
}

\author{
François Hugonnier
}

Belin | Revue française d'études américaines

$2012 / 3-n^{\circ} 133$

pages 38 à 52

ISSN 0397-7870

Article disponible en ligne à l'adresse:

http://www.cairn.info/revue-francaise-d-etudes-americaines-2012-3-page-38.htm

Pour citer cet article :

Hugonnier François, « L'artiste faussaire et la création du réel : The Brooklyn Follies de Paul Auster », Revue française d'études américaines, 2012/3 n 133, p. 38-52.

Distribution électronique Cairn.info pour Belin.

(c) Belin. Tous droits réservés pour tous pays.

La reproduction ou représentation de cet article, notamment par photocopie, n'est autorisée que dans les limites des conditions générales d'utilisation du site ou, le cas échéant, des conditions générales de la licence souscrite par votre établissement. Toute autre reproduction ou représentation, en tout ou partie, sous quelque forme et de quelque manière que ce soit, est interdite sauf accord préalable et écrit de l'éditeur, en dehors des cas prévus par la législation en vigueur en France. II est précisé que son stockage dans une base de données est également interdit. 


\title{
L'artiste faussaire et la création du réel : The Brooklyn Follies de Paul Auster
}

\author{
François HUGONNIER
}

\begin{tabular}{|c|}
\hline mots-clés/key-words \\
$\frac{\text { indicible; }}{\text { pharmakon; représentation; }}$ \\
reproduction; 11 septembre; \\
témoignage; \\
hyperréalité \\
$*$ \\
unspeakable; \\
pharmakon; representation; \\
reproduction; $9 / 11 ;$ \\
testimony; \\
hyperreality
\end{tabular}

Since his debut as a poet, Paul Auster has pursued his metaphysical investigations in a semiotic world where signs are everywhere to be read, where doubts about authenticity and forgery are ubiquitous. In The Brooklyn Follies (2005), the multiplicity of originals and facsimiles, the silence/speech duality and the architectural metaphors lead to the final vision of the attacks on the World Trade Center. As the reminiscences of the confusio linguarum contribute to the novel's plot, Paul Auster represents 9/11 "obliquely" and reaffirms the role of language and the imagination in the rebuilding of truth.

$\mathrm{N}_{\mathrm{t}}$ athan Glass, le personnage principal de The Brooklyn Follies, témoigne de façon extrêmement concise et inattendue de la chute des tours du WTC. Ce roman est représentatif de l'œuvre post-11 septembre de Paul Auster, qui offre un lieu d'inscription du témoignage en empruntant des voies détournées. La prose musicale et « indirecte » (NPR, 2002) de ce texte à la surface lisse et légère dissimule pendant plus de trois cents pages l'événement traumatique qui ne surgit que dans les dernières lignes. La structure de cet ouvrage à la fois pré et post-11 septembre est en ce sens révélatrice de « l'écriture du trauma » qui est « toujours mémoire vive d'une mort à venir » (Amfreville 195). The Brooklyn Follies soulève un ensemble de questions relatives aux modes du témoignage, à l'interaction entre perception et représentation, réalité et fiction. 
La vision traumatique (witness) n'est dévoilée qu'au terme du processus d'écriture (testimony) entrepris par Nathan. Déconstruisant ses propres chimères architecturales, le narrateur écrivain chemine pas à pas, d'une nuance de silence à une autre, vers cette image finale. L'absence des tours infiltre les espaces vides du texte qui s'en trouvent saturés, contaminant les personnages par une aphasie pré-traumatique maladive et proleptique. Entre détours de silence et silence des tours, les thèmes du langage (contrefaçon et pharmakon), de la reproduction et de l'hyperréalité exigent une mise en perspective de The Brooklyn Follies avec l'ensemble de l'œuvre de Paul Auster. Afin d'éviter les «pièges de surface » (Auster, 2005a 222) de ce roman relativement peu étudié dont la superficialité masque une profondeur symbolique, une analyse critique conséquente s'avère nécessaire.

\section{Le langage ou l'art de la contrefaçon}

Dans son petit traité de métaphysique White Spaces (1980), qu'il considère rétrospectivement comme une passerelle entre poésie et prose (« the bridge between writing poetry and writing prose » [Auster, 1995 132]), Paul Auster affirme le rôle de l'imagination dans la recherche de la vérité : «At any given moment I find myself on the brink of discovering some terrible, unimagined truth » (Auster, 2004a 159, je souligne). Il précise également que le langage est une contrefaçon de la réalité : « words falsify the things they attempt to say, but even to say "they falsify" is to admit that "they falsify" is true, thus betraying an implicit faith in the power of words to say what they mean to say » (158). L'œuvre d'Auster est façonnée de langage et forgée de silence. La crise et la quête de sens commencent avec la confusio linguarum, depuis ses premiers écrits d'écolier (datés de 1967) jusque dans son œuvre de fiction en passant par son œuvre poétique de jeunesse (1970-1979). Auteur juif-américain séculier, héritier du poststructuralisme et des poètes objectivistes - dont il se distingue par son doute viscéral sur la validité du matériau linguistique ${ }^{1}-$, Paul Auster produit des romans qui portent les traces d'une réflexivité participant à la création d'un monde où tout fait signe, où tout renvoie à une construction linguistique seconde, où réalité et fiction se confondent.

Paul Auster a définitivement mis fin à son activité poétique face à l' « impossibilité des mots », le langage trahissant son rapport au monde. Si l'œil du poète perçoit et intériorise le monde dont il fait partie (« The world is in my head. My body is in the world » [« Notes from a Composition Book, 1967 », Auster, 2004a 203]), le langage est un medium inadéquat, le marqueur tangible - une fois le signifié re-présenté par le destinataire - d'une vérité perdue dès lors que le mot est prononcé ou écrit sur la page blanche. Auster trouve sa vérité subjective dans l'enfermement linguistique, comme le résume la dernière strophe du poème «Interior » : «In the impossibility of words, / in 
the unspoken word that asphyxiates, / I find myself » (Auster, 2004a 69). Le chant poétique austérien déplore une univocité souffrante et invite au silence. Comprenant que l'utilisation de « je » est la première étape de son enfermement linguistique (il s'agit bien là de « l'invention de la solitude »), Auster mesure la portée du « je est un autre » rimbaldien (Rimbaud 84) et trouve sa voie dans l'imagination dialogique et la désubjectivation. Avec la multiplicité des voix narratives souvent paradoxales de ses personnages écrivains ou conteurs, son œuvre romanesque s'inscrit dans la tradition de Walt Whitman : «I am large, I contain multitudes » (« Song of Myself» [Whitman 176]). Mark Brown souligne le fait que le combat poétique avec le langage reste un élément central dans les romans de Paul Auster : «the struggle with language has remained in his novels » (Brown, np). Comme l'illustre The Brooklyn Follies, le passage de la poésie à la fiction n'est pas une rupture, mais une continuité.

Poursuivant les questionnements fondamentaux de son premier roman City of Glass (The New York Trilogy [Auster, 1987]), The Brooklyn Follies est construit à partir d'une dichotomie linguistique aux multiples ramifications. Dès l'introduction - «I was looking for a quiet place to die » (Auster, 2005a 1; je souligne) - le texte en prose est généré par la confrontation silence/parole renvoyant à Babel, à la recherche de la langue parfaite et au logocentrisme. Le personnage central de Lucy est une fillette refusant obstinément de parler (son prénom évoque la lumière mystique). Elle accède à un langage non-verbal paradoxalement moins stérile que les discussions extensives entre son oncle Tom Wood et son grand oncle Nathan Glass. Grâce à son silence, Lucy parvient à faire ressentir clairement ses émotions, alors que Nathan et Tom n'arrivent à rien en utilisant le langage :

I had been hoping to trick a few words out of her, but all I got were the same nods and shakes that Tom had been subjected to earlier. Strange unsettling little person. [...] She looked you straight in the eye, understood everything you said. [...] Tom and I withdrew to the kitchen, where she wouldn't be able to overhear our conversation. We talked for a good thirty or forty minutes, but nothing came of it except ever-mounting confusion and worry. [...] Round and round we went, the two of us traveling in circles, talking, talking, talking, but unable to answer a single question.

La clef de l'énigme réside dans le fait que Lucy et sa mère Aurora sont victimes du Révérend Bob, qui contraint les familles au silence intérieur afin de retrouver la langue originelle de la Création :

Our church. The Temple of the Holy Word. We're a small group. Our congregation has just sixty members, but the Reverend Bob is an inspired leader, and he's taught us many things. "In the beginning was the Word, and the Word was with God, and the Word was God». [...] He understands what it means to sacrifice. If the Word is God, then the words of men mean nothing. They're no more significant than the 


\section{L'ARTISTE FAUSSAIRE ET LA CRÉATION DU RÉEL...}

grunts of animals or the cries of birds. To breathe God into us and absorb His Word, the reverend instructs us to refrain from indulging in the vanity of human speech. That's the sacrifice. One day out of seven, every member of the congregation must maintain a full and unbroken silence for twenty-four straight hours.

$(251-252)$

Le Révérend Bob n'est pas le premier linguiste fou rencontré dans les essais critiques (voir l'article « New York Babel» sur Le Schizo et les langues de Louis Wolfson [Auster, 2003 325-330]) ou dans l'œuvre de fiction de Paul Auster (dans City of Glass, Peter Stillman est un docteur dément qui enferme son fils Peter Stillman et le réduit au silence afin de retrouver la langue pure de la Création - The Word of God). La Genèse, et plus particulièrement la parabole de la Tour de Babel, sont des leitmotivs incontournables dans les poèmes de Paul Auster, puis dans de nombreux romans, comme ici dans The Brooklyn Follies. Paul Auster évolue dans un univers sémiotique où le doute entre original et contrefaçon est intrinsèque à toute investigation métaphysique. La question récurrente est alors celle de la formulation de l'indicible. Dans The Brooklyn Follies, Auster fait d'ailleurs une allusion discrète à Wittgenstein qui «montre ${ }^{2}$ » ce qui ne peut être dit. Les enseignements de Wittgenstein sont appliqués ironiquement par Auster : Nathan Glass raconte une anecdote de la vie du philosophe sans citer la célèbre injonction qui conclut le Tractatus logico-philosophicus (1922). Auster ne parle pas de l'indicible qu'il met en scène, mais le montre.

Parallèlement à cette dualité entre parole et silence le roman est construit autour de la confrontation objet/signe linguistique, et par extension original/contrefaçon. L'intrigue est fondée sur l'acquisition d'une contrefaçon du manuscrit de The Scarlet Letter qui s'avère finalement inexistante : « the Hawthorne scam was no more than a ruse, an elaborate hoax within a hoax » (Auster, 2005a 210). De nombreux éléments du récit font écho à ce faux, comme par exemple la folle recherche de la langue originelle (le Révérend Bob est qualifié de «fraud » et de «scam artist from the word go » [263]). On soulignera aussi la présence de personnages artistes faussaires qui produisent des copies de tableaux plus authentiques que les originaux (le duo Harry Brightman et Alec Smith). Les thèmes de la contrefaçon, du faussaire, de l'authenticité et de la reproduction évoquent des écrivains tels qu'André Gide, Walter Benjamin, Jorge Luis Borges ${ }^{3}$, Jean Baudrillard, ou plus récemment Umberto Eco (Le Cimetière de Prague, 2011). Mais la ressemblance la plus frappante avec The Brooklyn Follies est The Recognitions de William Gaddis (1955), auteur américain cité, semble-t-il, au hasard des rayonnages de «Brightman's Attic », la librairie d'éditions originales tenue par Harry Brightman (Auster, 2005a 57).

Comme dans The Brooklyn Follies, au centre de l'intrigue de The Recognitions se trouvent des faussaires, et notamment Wyatt Gwyon, le per- 
sonnage principal. D'après Brigitte Félix, son « immense talent lui permet la production de faux si parfaits qu'ils sont indétectables. Ainsi peuvent être simultanément posées les questions de la religion, de la valeur, de l'origine, et de l'art » (Félix 21). Paul Auster reprend l'image structurante de ce récit dans The Brooklyn Follies. The Recognitions est en quelque sorte la reproduction d'un ouvrage qui est lui-même probablement une contrefaçon : «Les Reconnaissances est la traduction anglaise du titre latin (Recognitiones), des Reconnaissances clémentines, un texte dont l'origine est problématique. On n'en connaît pas l'auteur » (Félix 22). Le titre du livre de William Gaddis est donc un "emprunt non signalé », une « non-reconnaissance de dette » (23). En faisant discrètement référence à William Gaddis dans la librairie d'Harry Brightman, Paul Auster met en abyme un hypotexte important de The Brooklyn Follies. Le roman de Paul Auster n'est qu'une contrefaçon supplémentaire, une réécriture. L'élan créatif originel est insondable. Le texte, qui est une construction linguistique peu fiable, ne parviendra à en communiquer qu'une image évanescente.

\section{La pharmacie de Nathan}

Dans The Brooklyn Follies, la régression vers l'origine - qu'il s'agisse d'authenticité artistique ou de filiation - est rendue impossible par la propagation des fac-similés, ce qui a pour ultime conséquence d'empêcher toute distinction entre le vrai et le faux, c'est-à-dire entre des originaux systématiquement absents et des contrefaçons trop présentes. Cette remarque d'ordre générique s'applique à l'ensemble du roman : le langage y implique toujours un manque et un excès de sens.

La réappropriation du thème de l'ambiguïté sexuelle (Harry Brightman et Tina Hott) est particulièrement représentative de ce phénomène. Dans The Brooklyn Follies, la contrefaçon prend la forme du travestisme. Il s'agit aussi d'un thème central de The Recognitions, comme l'explique Brigitte Félix : « Le monde de l'ambiguïté sexuelle est associé à celui de l'argent et de la contrefaçon réunis dans le mot queer, qui désigne la fausse monnaie aussi bien que l'homosexuel en argot. Le monde de l'argent et celui du faux sont intimement liés dans le texte à la question de l'identité sexuelle et de la recherche du père » (Félix 39). Suite au décès d'Harry Brightman, le personnage transsexuel Tina Hott - fac-similé par excellence - interpelle Nathan lors de la cérémonie funèbre. Il ne peut plus distinguer le « vrai » (Rufus) du « faux » (Tina Hott) :

He was one of the most beautiful women I had ever seen. Decked out in full widow's regalia, with a tight black dress, three-inch black heels, and a black pillbox hat with a delicate black veil, he had turned himself into an incarnation of absolute femininity, an idea of the feminine that surpassed anything that existed in the realm of natural womanhood. The auburn wig looked like real hair; the 


\section{L'ARTISTE FAUSSAIRE ET LA CRÉATION DU RÉEL...}

breasts looked like real breasts; the makeup had been applied with skill and precision; and Tina's legs were so long and lovely to look at, it was impossible to believe that they were attached to a man.

But there was more to the effect she created than mere surface trappings. More than just clothes or wigs or makeup.

(Auster, 2005a 222; je souligne)

Dans cette description de l'incarnation « transcendante » (223) de l'art le plus pur, dans cette personnification de la recherche de l'inspiration ultime de tout romancier (« inner light »), Paul Auster suggère d'aller rechercher un sens plus profond, de ne pas être aveuglé par le masque de l'illusion romanesque, ni par sa prose fluide, trop évidente et superficielle (« surface trappings », «makeup »). Nathan Glass marque radicalement la saisissante transformation de Rufus par le changement de pronom personnel et l'amorce d'un nouveau paragraphe. La métamorphose n'arrive à son terme que lorsque Nathan atteste qu'il s'agit de la plus belle femme qu'il ait jamais vue. L'utilisation soudaine du pronom personnel féminin (« she ») renforce cette rupture en accomplissant littéralement le changement de sexe. Le faux surpasse l'original et Tina incarne la féminité absolue ( «absolute femininity »). La contrefaçon devient plus réelle que l'original. Le mot « real » est d'ailleurs utilisé deux fois au cours de cette description, pour qualifier la perruque (« real hair ») et la poitrine de Tina (« real breasts »), ce qui témoigne de l'illusion d'un faux «plus réel » que le vrai.

Dans The Brooklyn Follies, comme dans The Recognitions, il apparait, pour reprendre la formule de Marc Chénetier, que

les amonts sont irréductibles, [...] il n'est pas d'original, mais toujours et à jamais des copies et des redites, [...] il n'y a, dirait Derrida après Socrate, que du pharmakon, du "supplément », de l'ajout empoisonné ; toute imitation ou répétition en aval est déjà source de tromperie sur la marchandise ; il devient de plus ontologiquement impossible de distinguer le faux du vrai.

(Chénetier 45)

Le mot grec pharmakon peut signifier « remède » ou « poison ». Lors de sa déconstruction du pharmakon de Platon, Jacques Derrida précise que « [s]i le pharmakon est « ambivalent », c'est donc bien pour constituer le milieu dans lequel s'opposent les opposés, le mouvement et le jeu qui les rapportent l'un à l'autre, les renverse [sic] et les fait [sic] passer l'un dans l'autre (âme/corps, bien/mal, dedans/dehors, mémoire/oubli, parole/écriture, etc.) » (Derrida 158). C'est avec une telle ambivalence que Paul Auster aborde les contrefaçons et le langage dans The Brooklyn Follies. Toute une constellation de termes dichotomiques est soumise à ce « renversement ». À la fin du roman, quelques heures avant les attentats du World Trade Center, Nathan s'approche précisément d'un espace de réconciliation des opposés. Sur son lit d'hôpital, il fait ce qui s'apparente à une expérience de mort imminente, dont il témoigne comme suit : 


\section{FRANÇOIS HUGONNIER}

I was in there with myself, rooting around with a kind of scrambled desperation, but I was also far away, floating above the bed, above the ceiling, above the roof of the hospital. I know it doesn't make any sense, but lying in that boxed-in enclosure with the beeping machines and the wires clamped to my skin was the closest I have come to being nowhere, to being inside myself and outside myself at the same time.

(Auster, 2005a 297)

Ce passage est extrait de l'avant-dernier chapitre de The Brooklyn Follies, intitulé «Inspiration ». Auster rend ici hommage à Maurice Blanchot, dont «L'inspiration », chapitre phare de L'espace littéraire, traite des limites de l'expérience. Blanchot compare le domaine de la mort à celui de la vérité ultime et inaccessible qu'est le centre attracteur de l'œuvre (symbolisé aussi par le « dehors », la « nuit » et le regard d'Orphée). Dans le chapitre «Inspiration », Nathan Glass semble accéder à ce que Maurice Blanchot nomme « l'Ouvert»:

l'Ouvert, la libération de la parole orphique en qui s'affirme l'espace, l'espace qui est un «Nulle part sans nom ». Parler est alors une transparence glorieuse. Parler n'est plus dire, ni nommer. Parler, c'est célébrer, et célébrer, c'est glorifier, faire de la parole une pure consumation rayonnante qui dit encore qu'il n'y a plus rien à dire, qui ne donne pas un nom à ce qui est sans nom, mais l'accueille, l'invoque et le célèbre.

(Blanchot, 1955 208-209)

Nathan Glass se situe dans un « Nulle part sans nom »: «being nowhere, being inside myself and outside myself at the same time » (Auster 2005a, 297) ; «I was no one » (300). Il « accueille » et « invoque » cet espace neutre, « au sens étymologique de ne-uter - "ni l'un ni l'autre” » (Killeen 36) : «Remarkably enough, I wasn't scared. [...] You merely accepted. You merely took what you were given, and if death was what I had been given that day, I was prepared to accept it » (Auster, 2005a 295).

C'est alors que se dessine le projet de Nathan Glass, qui consiste à publier les biographies d'inconnus afin de les célébrer (« to publish biographies of the ordinary, the unsung » [300]), à l'instar de Rilke, dont Blanchot cite les vers suivants :

O, dis-moi, poète, ce que tu fais. - Je célèbre.

Mais le mortel et le monstrueux,

comment l'endures-tu, l'accueilles-tu? - Je célèbre.

Mais le sans nom, l'anonyme,

comment, poète, l'invoques-tu cependant? - Je célèbre.

(Blanchot, 1955 209)

Paul Auster explique en effet que The Brooklyn Follies est avant tout un hymne aux gens ordinaires («a hymn to ordinary life » [La Clé des Langues, 2009]), « ceux-là », précise Blanchot, « [dont] il n'est plus entendu parler, ils ne laissent pas de carnet de route, ils n'ont pas de nom, anonymes dans la 
foule anonyme » (Blanchot, 1955 224). Nathan Glass veut « ressusciter » les hommes sans particularités à l'aide des mots : «I would resurect that person in words » (Auster, 2005a 302). Il conclut ce moment d'inspiration mystique avec une phrase clef, qu'Auster qualifie lui-même d'un mélange de sérieux et d'humour « tongue-in-cheek » (La Clé des Langues) : " one should never underestimate the power of books » (Auster, 2005a 302). Mêlant spiritualité et jeux intertextuels complexes à une tonalité superficielle, voire mièvre, relevant du pastiche, Paul Auster s'affirme donc dans une écriture ambiguë à la rencontre des opposés. Nathan Glass le confirme avec cette conclusion sur la prestation silencieuse de Tina Hott en « faux singer » (222) : «It was magnificent and absurd. It was funny and heartbreaking. It was moving and comical. It was everything it was and everything it wasn't » (223).

\section{L'ère de la reproduction et la création du réel}

Lors de la cérémonie funèbre organisée à la mémoire d'Harry Brightman, Tina Hott incarne le franchissement réussi de la frontière entre original et contrefaçon, parole et silence, réalité et fiction. Ce personnage participe aussi à une réflexion d'envergure sur la problématique de l'hyperréalité. La destruction des tours du World Trade Center survient à la fin du roman, créant une onde de choc silencieuse et soulevant d'autres questions complexes relatives à la représentation artistique et à la valeur de l'art. Comme l'affirme Walter Benjamin, « dès lors que le critère d'authenticité n'est plus applicable à la production artistique, toute la fonction de l'art se trouve bouleversée » (Benjamin 282). Un «bouleversement » épistémologique et artistique est le point de départ et la ligne d'horizon de The Brooklyn Follies.

Nul ne peut se repérer dans la foule des contrefaçons, des personnages anonymes qui hantent les pages de ce roman de l'ère de la reproduction. Lors d'une discussion sur les grands écrivains morts dans l'anonymat, Tom fait allusion à Musil (Auster, 2005a 149) et son gigantesque roman inachevé L'homme sans qualités $(1930,1932)$. Dans son étude sur Musil, Maurice Blanchot définit l'homme sans qualités comme «l'homme quelconque des grandes villes, l'homme interchangeable, qui n'est rien et n'a l'air de rien, le "On" quotidien, l'individu qui n'est plus un particulier, mais se confond avec la vérité glaçante de l'existence impersonnelle »(Blanchot, 1959 192). The Brooklyn Follies est peuplé de personnages qui ne sauraient trouver leur vérité, leur origine et leur originalité, leur singularité. L' « ersatz James Joyce » (Auster, 2005a 221), par exemple, évoque l'opposition entre l'original (le célèbre écrivain irlandais) et la contrefaçon (le personnage insignifiant portant le même nom dans The Brooklyn Follies). Autre personnage signifiant par son insignifiance, Al Wilson explique que son nom relève de l'anonymat: "'I've been Al Wilson since the day I was born which is maybe half a step up from being called John Doe. [...] 
There must be a thousand Al Wilsons in Vermont alone" » (163). Comme Auster invite constamment aux jeux paronomastiques, augmentés de l'omniprésence de doppelgängers, un rapprochement s'opère entre $\mathrm{Al}$ Wilson, l'homme aux multiples contrefaçons, et Alec Smith, jeune peintre prodige, authentique et mystérieux, dont l'œuvre est plagiée suite à son décès accidentel (en d'autres termes, Al Ex-Myth). D'autres clins d'œil furtifs, comme par exemple la séance de cinéma avec Modern Times de Chaplin (141) contribuent à ce tableau silencieux de l'existence impersonnelle en série.

Élément crucial du paradigme de l'anonymat, la tombe anonyme ( « unmarked grave ») est un motif récurent lors des discussions entre Nathan, Tom et Aurora $(150,273)$. Bien avant les événements historiques du 11 septembre 2001 qui surgissent à la fin de The Brooklyn Follies, ces conversations rappellent l'histoire personnelle d'Auster et la mort soudaine de son père, véritable déclencheur de son œuvre en prose. Le poème « S. A. 1911-1979 » (Auster, 2004a 144), par son silence et son titre portant simplement les initiales de Samuel Auster, est évocateur d'une pierre tombale presque anonyme. La vie et la mort du père d'Auster, un « homme invisible », sont étudiées plus longuement dans son mémoire en prose « Portrait of an Invisible Man » : «I did not shed any tears. [...] In some strange way, I was remarkably prepared to accept this death, in spite of its suddenness. What disturbed me was something else, something unrelated to death or my response to it: the realization that my father had left no traces » (Auster, 1988 6). À la fin de The Brooklyn Follies, cette réflexion semble refaire surface et se poursuivre avec le projet de publication des biographies d'inconnus élaboré par Nathan le 10 septembre 2001 : «Most lives vanish. A person dies, and little by little all traces of that life disappear. An inventor survives in his inventions, an architect survives in his buildings, but most people leave behind no monuments or lasting achievements » (Auster, 2005a 301). En observant attentivement cette phrase on comprend que l'expression « unmarked grave » et la question de la mort anonyme sont associées aux métaphores architecturales (« an architect survives in his buildings », « no monuments » [301]). Ces réseaux de signification souterrains sont clairement dévoilés - montrés, pour reprendre Wittgenstein - lorsque la «marque » apparaît enfin dans le titre du dernier chapitre, «X Marks the Spot» (303; je souligne). Il s'agit de l'unique extrait de l'œuvre romanesque d'Auster témoignant directement ${ }^{4}$, bien que furtivement, de la chute des tours du World Trade Center :

It was eight o'clock when I stepped out onto the street, eight o'clock on the morning of September 11, 2001-just forty-six minutes before the first plane crashed into the North Tower of the World Trade Center. Just two hours after that, the smoke of three thousand incinerated bodies would drift over toward Brooklyn and come pouring down on us in a white cloud of ashes and death. 
Paul Auster suggère que Ground Zero est une fosse commune (mass grave). Lorsque Nathan témoigne de la chute des tours, il parle de « the smoke of three thousand incinerated bodies » (304, je souligne). Cette allusion discrète à Auschwitz est une répétition exacte, mais incomplète, des termes employés par Auster à l'occasion de sa préface pour le catalogue d'une exposition d'Art Spiegelman, parue deux ans plus tôt : «Then came September 11,2001 . In the fire and smoke of three thousand incinerated bodies, a holocaust was visited upon us » (Auster 2003, 462). Paul Auster considère donc le 11 septembre comme la re-présentation du désastre. Il s'agit d'une nouvelle occurrence, comme l'illustrent l'utilisation de l'article indéfini «a » et l'absence de majuscule à « holocaust». Le terme « holocaust » est utilisé en tant que métonymie représentative du désastre à une échelle universelle. Exprimant brièvement sa perception des attentats du World Trade Center, Auster a recours à un autre événement historique pour parler d'un fait qui dépasse l'entendement, dévoilant son incapacité à cerner la vérité de ce drame singulier et sans précédent. Paul Auster a même finalement opté pour une expression elliptique dans The Brooklyn Follies, où la phrase répétée par Nathan Glass ne comporte plus les termes « fire » ni « holocaust ». Cette question indicible ne peut être abordée par le langage, mais bien par l'art qui évolue en dehors du langage et franchit les limites du dicible, de l'imaginaire et de la représentation. Dans cet essai sur Art Spiegelman, Auster décrit d'ailleurs la célèbre couverture du magazine The New Yorker (24 septembre 2001) un « noir sur noir », une « anti-image » des tours qu'il reconnaît comme étant son chef d'œuvre ${ }^{5}$.

Dans The Brooklyn Follies, les diverses métaphores architecturales jouent un rôle proleptique et sont étroitement liées à la vision finale des attentats du World Trade Center. Il faut bien sûr mentionner l' "Hotel Existence » (refuge utopique inventé par Harry, Tom et Nathan, associé à Walden de Thoreau), ou même le titre de l'œuvre, puisque l'un des sens de « folly » fait référence à un bâtiment d'ornement, une imitation. Ces édifices sont le fruit de l'imagination et d'une construction chimérique, tout comme le livre de Paul Auster qui n'est fait que de langage, un langage trompeur, système sémiotique à jamais second, simple représentation du monde. Pourtant, bien qu'il provienne d'un personnage de fiction, le témoignage final de Nathan est à la fois image de la réalité, et réalité de l'image.

Comme le laisse entendre The Brooklyn Follies par son silence, après la chute de la tour de Babel et la confusion des langues, les événements du 11 septembre sont le traumatisme de l'ère de l'hyperréalité. Dans l'œuvre post-11 septembre d'Auster, la chute des tours du WTC, entrant en résonance avec la Shoah en tant que «délocalis[ation du] traumatisme » (Angel-Perez 19), ainsi qu'avec Babel d'un point de vue strictement linguistique, est élevée au rang de nouveau récit fondateur. La destruction des tours jumelles à la fin du roman 
conduit à réfléchir à leur portée symbolique dans les thématiques des doubles, de la contrefaçon, de la reproduction et du langage. Dans les années soixantedix, Jean Baudrillard théorisait l'ère de la reproduction et « la fin de la représentation $^{6} »$, insistant sur la contemplation mutuelle des tours dans leur supériorité gémellaire. Selon Baudrillard, les tours ont court-circuité la compétition internationale en véhiculant un double symbole d'ascension hégémonique (Baudrillard, 1976 108-109). Comme l'affirme par ailleurs Maurice Blanchot, " [1]à où il y a un double parfait, l'original est effacé, et même l'origine » (Blanchot, 1959 133). D'après la Genèse, Dieu a puni les hommes pour leur arrogance, eux qui construisaient la tour de Babel pour atteindre le ciel. Les extrémistes djihadistes ont-ils vu dans la destruction des célèbres gratte-ciels (skyscrapers) une punition similaire ? En plaçant les réminiscences de Babel au centre de l'intrigue de ce roman qui se termine par l'évocation directe du 11 septembre 2001, Paul Auster sous-entend de telles considérations.

Dans son article «L'esprit du terrorisme », convoquant Jorge Luis Borges comme le ferait Auster, Baudrillard avance que la chute des tours jumelles est une «fiction au-delà de la fiction » (Baudrillard, 2001). Auster a souvent constaté que la réalité est bien plus surprenante et effrayante que la fiction : " reality is far more terrible than anything we can imagine ", explique-t-il à Joseph Mallia (Auster, 1995 115). Une réflexion sur les interactions entre réalité et fiction est absolument essentielle pour aborder le 11 septembre de manière « détournée » ou « indirecte » dans une œuvre littéraire, comme Auster le précise en faisant référence à Wallace Stevens dans l'émission diffusée sur NPR le 8 septembre 2002. Dans l'introduction du recueil True Tales of American Life, édité en partenariat avec NPR, Auster fait une remarque complémentaire au sujet des histoires vraies qu'il a collectées et éditées : "What interested me most, I said, were [...] true stories that sounded like fiction » (Auster, 2001 xiv). Le WTC, avant, pendant et après sa chute, cristallise la fascination d'Auster pour les événements réels dépassant l'entendement et l'imaginaire.

Souvent célébré comme un écrivain du hasard et des connexions étranges, Paul Auster s'amuse à effacer la frontière entre réalité et fiction, en employant la métalepse dans ses romans (City of Glass et Oracle Night [2004b] par exemple). Sa singularité artistique est construite sur le questionnement des signes dans l'élaboration d'un sens fuyant. Comme le préconisait déjà Auster à l'aube de sa carrière, bien avant que son ami funambule Philippe Petit n'effectue sa traversée aérienne entre les tours, que son personnage de fiction Peter Stillman ne se jette du pont de Brooklyn et que les authentiques falling men ne sautent des tours du World Trade Center, la chute originelle de l'homme a eu lieu "de l'œil à la bouche », dans le mouvement de perception et de représentation du monde : «The fall of man is not a question of sin, transgression, or moral turpitude. It is a question of language con- 
quering experience: the fall of the world into the word, experience descending from the eye to the mouth. A distance of about three inches » (« Notes from a Composition Book, $1967 »$ [Auster 2004a, 204]).

Face à la question posée par le nouveau traumatisme du 11 septembre, Auster réaffirme, par le témoignage personnel ${ }^{7}$ et la fiction, le rôle du langage et de l'imagination dans la reconstruction de la vérité. Dans The Brooklyn Follies les multiples romans (originaux, fac-similés, emprunts intertextuels) et les récits enchâssés forment une boîte à outils à cet effet. La fiction est alors - comme Auster le laisse entrevoir lorsque Tom raconte l'épisode de la poupée de Kafka (Auster 2005a, 152-155) -, un mensonge qui permet de dire la vérité.

L'une des vérités fondamentales de l'œuvre de Paul Auster, c'est que dire est une «impossibilité», car faire confiance aux pouvoirs du langage signifie jouer au faussaire. Paradoxalement, cet interdit de la représentation génère une aphasie constructive qui place le langage, l'écrivain témoin et le lecteur au cœur de la création du réel.

François Hugonnier est docteur en littérature américaine. Sa recherche porte sur «Les interdits de la représentation dans les œuvres de Paul Auster et Jérôme Rothenberg» (Université de Paris Ouest Nanterre La Défense). Auteur de plusieurs articles et entretiens consacrés à ces écrivains américains séculiers, il s'interesse aux formes hybrides entre poésie et prose, et plus particulièrement aux ruptures engendrées par les événements traumatiques d'Auschwitz et du 11 septembre 2001.

\section{OUVRAGES CITÉS}

Amfreville, Marc. Écrits en souffrance. Figures du trauma dans la littérature nord-américaine. Paris: Michel Houdiard, 2009.

Angel-Perez, Élisabeth. Voyages au bout du possible. Les théâtres du traumatisme de Samuel Beckett à Sarah Kane. Langres, Paris : Klincksieck, 2006.
Auster, Paul. White Spaces. Barrytown, NY : Station Hill, 1980.

-. The New York Trilogy. Londres:

Faber and Faber, 1987.

-. The Invention of Solitude. Londres :

Faber and Faber, 1988 [1982].

-. The Music of Chance. Londres:

Faber and Faber, 1991. 
-. The Red Notebook.

Londres : Faber and Faber, 1995.

- . True Tales of American Life.

Londres: Faber and Faber, 2001.

-. Collected Prose (Autobiographical

Writings, True Stories, Critical Essays,

Prefaces and Collaborations with

Artists). New York: Picador/Henry

Holt, 2003.

-. Collected Poems. Woodstock,

NY : One Overlook Press, 2004a.

-. Oracle Night. Londres: Faber

and Faber, 2004b.

-. The Brooklyn Follies.

Londres: Faber and Faber, 2005a.

-. Manhattan, Ground Zero :

A Sonic Memorial Sound Walk.

New York: Soundwalk, 2005b.

-. Man in the Dark. Londres: Faber and Faber, 2008.

-. Invisible. New York:

Henry Holt, 2009.

-. Sunset Park. New York:

Henry Holt, 2010.

BAUdRILlaRd, Jean. L'Échange

symbolique et la mort.

Paris: Gallimard, 1976.

—. «L'Esprit du terrorisme»,

Le Monde. 2 novembre 2001.

BENJAMIN, Walter. «L'CEuvre d'art à l'époque de sa reproductibilité technique» (1935-1939). CEuvres III. Paris : Gallimard, 2000.

Blanchot, Maurice. L'Espace littéraire. Paris : Gallimard, 1955.

—. Le Livre à venir. Paris: Gallimard, 1959.

BorGES, Jorge Luis. «La Bibliothèque de Babel». Fictions. Paris: Gallimard, 1956.

BROWN, Mark. Discours d'introduction prononcé lors du colloque «The New
York Trilogy and the American

Metropolis ». University

of Northampton. June 29, 2012.

Celan, Paul. Choix de poèmes réunis par l'auteur. Traduction et présentation de Jean-Pierre Lefebvre. Édition bilingue. Paris: Gallimard, [1968], 1998.

ChÉnetier, Marc. Au-delà du soupçon. Paris: Seuil, 1989.

Derrida, Jacques. La Dissémination. Paris : Seuil, 1972.

Eco, Umberto. Le Cimetière de Prague. Paris: Grasset, 2011.

FÉLIX, Brigitte. William Gaddis, l'alchimie de l'écriture.

Paris : Belin, 1997.

FINKELSTEIN, Norman.

«In the Realm of the Naked Eye.

The Poetry of Paul Auster».

Éd. Dennis Barone. Beyond The Red

Notebook. Philadelphie:

U of Pennsylvania P, 1995. 44-59.

GADDIS, William. The Recognitions. New York: Penguin, 1993 [1955].

GIDE, André. Les Faux-monnayeurs. Paris : Gallimard, 1997 [1925].

HaWthorne, Nathaniel. The Scarlet Letter. Londres: Penguin Popular Classics, 1994 [1850].

HugonNiER, François. «Speaking the Unspeakable: Auster's Semiotic World». Éds Stefania Ciocia et Jesús A. González. The Invention of Illusions: International Perspectives on Paul Auster. Newcastle:

Cambridge SP, 2011. 259-287.

KILLEEN, Marie-Chantal.

Essai sur l'indicible. Jabès,

Duras, Blanchot.

Saint-Denis : PU de Vincennes, 2004. 


\section{L'ARTISTE FAUSSAIRE ET LA CRÉATION DU RÉEL...}

La Clé des Langues, ENS Lyon.

"The "Mechanics of Reality":

Paul Auster Speaks about His Work and Inspiration ». The Brooklyn Follies and $9 / 11.13$ janvier 2009. $<$ http://cle.ens-lyon.fr/39808285/0/ fiche__pagelibre/> (consulté le 16 avril 2010).

NPR. «Authors Paul Auster and Salman Rushdie reflect on September $11 »$. All Things Considered. National Public Radio. September 8, 2002.

Rimbaud, Arthur. Poésies. Une saison en enfer. Illuminations. Paris : Gallimard, 1999.
Thoreau, Henry David. Walden. Londres: Dent, Everyman's Library, 1965 [1854].

Whitman, Walt. Leaves of Grass. Introduction, traduction et notes par Roger Asselineau. Édition bilingue. Paris : Aubier-Flammarion, 1972.

WitTGENSTEIN, Ludwig. Tractatus logico-philosophicus. Traduction, préambule et notes de Gilles-Gaston Granger, introduction par Bertrand Russell. Paris: Gallimard, 1993 [1922].

Wolfson, Louis. Le Schizo et les langues. Préface de Gilles Deleuze. Paris: Gallimard, 1970.

\section{NOTES}

1. Comparant le mouvement objectiviste et l'œuvre poétique d'Auster, Norman Finkelstein fait la remarque suivante : « all the reassuring materials of the objectivist lyric, quietly celebrated for their mere being - are gone » (Finkelstein 53). Finkelstein parle aussi de « resolute unmaking » (53). Il me semble que cette caractéristique de l'œuvre poétique d'Auster le rapproche davantage d'écrivains comme Edmond Jabès ou Paul Celan. Jean-Pierre Lefebvre insiste en effet sur les «"négations" celaniennes » (Celan 18). On peut tout autant parler de « négations » austériennes (que j'ai appelé ailleurs « unwords » [Hugonnier, 2011 271, 284285]). Dans l'œuvre de Celan, le mot « schaufeln, manier la pelle », signifie « creuser des fosses : écrire. Remplir des pages, écrire : creuser, vider » (Celan 10). D’après Lefebvre, « sa poétique se fonde là : donner le sens à la parole (en allemand : l'éclairer), c'est creuser son néant, “donner son ombre" » (Celan 10). Dans la poétique d'Auster, écrire, parler, c'est creuser, mais surtout poser et manipuler des pierres, construire des murs. Utiliser des mots pour construire, déconstruire, s'enfermer. Voir le recueil « Disappearances » (Auster, 2004a 105-114) qui permet de mieux apprécier la portée de ces thèmes dans l'œuvre de fiction (The New York Trilogy, The Music of Chance [1991], Oracle Night [2004b], The Brooklyn Follies, Invisible [2009], etc.).

2. Wittgenstein affirme en effet qu' « [i]l y a assurément de l'indicible. Il se montre, c'est le Mystique » (Wittgenstein [6.522] 112). Dans The Brooklyn Follies, l' « anecdote » de la biographie de Wittgenstein ouvre la voie de l'interprétation à qui sait lire entre les lignes.

3. « Si chaque exemplaire est unique et irremplaçable, il y a toujours, la Bibliothèque étant totale, plusieurs centaines de milliers de fac-similés presque parfaits qui ne diffèrent du livre correct que par une lettre ou par une virgule » (Borges 165). 


\section{NOTES}

4. Les événements du 11 septembre sont systématiquement traités de façon détournée dans les autres romans, comme dans l'uchronique Man in the Dark (2008) par exemple.

5. Auster emploie également un mot-clef pour décrire cette œuvre de Spiegelman : « sublime » («Spiegelman had found his direction. Not in silence - but in the sublime » [Auster, 2003 463]). Le « sublime » et la «nature morte » sont au cœur de la problématique des interdits de la représentation liés au 11 septembre dans The Brooklyn Follies (2005), Man in the Dark (2008) et Sunset Park (2010).

6. «Tout Andy Warhol est là : les répliques multipliées du visage de Marilyn sont bien en même temps la mort de l'original et la fin de la représentation. Les deux tours du W.T.C. sont le signe visible de la clôture d'un système dans le vertige du redoublement. » (Baudrillard, 1976 108).

7. Voir «Random Notes-September 11, 2001-4:00 PM» et «NYC=USA» (Auster, 2003 505-506; 510). Auster a également prêté sa voix à l'enregistrement de Manhattan, Ground Zero : A Sonic Memorial Soundwalk (New York: Sound Walk [Auster 2005b]). 